\title{
Advances in Interventional Oncology: Percutaneous Therapies
}

\author{
Nadim Muallem $\cdot$ Stephen B. Solomon
}

Published online: 20 April 2014

(c) Springer Science+Business Media New York 2014

\begin{abstract}
The field of interventional oncology has emerged over the last decade to focus on the minimally invasive image-guided techniques for the treatment of cancer. Ablative techniques specifically have evolved in more recent years and garnered significant evidence supporting their increased inclusion in standards of care and their novel use as curative or disease-modifying therapies, while still maintaining a large role in supportive or symptomatic treatment. Percutaneous ablation will more than likely continue to evolve because of the parallel innovations in imaging and engineered medical devices used in percutaneous interventions but also the improved understanding of cancer behavior and development of personalized cancer therapy.
\end{abstract}

Keywords Radiofrequency ablation - Microwave ablation - Cryoablation - Lung cancer · Liver cancer . Kidney cancer

\section{Introduction}

This review of percutaneous interventions in cancer therapy will mainly focus on percutaneous ablation techniques that are commonly performed in thoracic and abdominal interventions on lung, liver and renal tumors with a small discussion on pancreatic tumors. These techniques rely on

This article is part of the Topical Collection on Essentials in Oncologic Imaging.

N. Muallem $(\bowtie) \cdot$ S. B. Solomon

Department of Radiology, Memorial Sloan-Kettering Cancer

Center, 1275 York Ave, New York, NY 10021, USA

e-mail: muallemn@mskcc.org image guidance for monitoring targeted tumor necrosis in a predetermined area and can be thermal or non-thermal mediated.

\section{Non-Thermal Ablation}

Non-thermal ablation consists of percutaneous injection of tumoricidal agents such as alcohol or acetic acid into the tumor. Though well established and still used in select cases, it has been largely supplanted by thermal techniques [1]. Irreversible electroporation (IRE) is another non-thermal ablation technique that relies on application of high-intensity electrical pulses through a target field inducing cellular disruption and necrosis. The lack of thermal effects in IRE is thought to preserve vascular and ductal structures within the treatment field since it does not affect the supporting connective tissue matrix [2]. Though used clinically with some success [3], it is still in its early stage with a paucity of publications over recent years, but trials are underway and may provide further evidence to support its use [4].

\section{Thermal Ablation}

Thermal Ablation Essentially Consists of Heating or Freezing of Target Tissues

Heat ablation relies on the principle of thermal damage and coagulation necrosis of tumor cells with irreversible tumor cell damage achieved within $4-6 \mathrm{~min}$ at $50-55^{\circ}$ and near immediate coagulation and cellular damage at $60-100^{\circ}$ [5].

Tissue heating is most commonly achieved through percutaneous needle applicators such as in radiofrequency (RF) ablation and microwave (MW) ablation. The former 
relies on the passage of electrical currents through tissue, while the latter generates an oscillating electromagnetic field causing friction of oscillating water molecules in the target. RF is the oldest and least expensive form of thermal ablative technology and has the highest experience profile among practitioners. The relatively small ablation zone in $\mathrm{RF}$ ablation limits the size of tumors eligible for treatment and often requires multiple ablation probes or repositioning, but it has also been thought to contribute to an increased safety profile and predictability. The disadvantage is that this may lead to longer treatment times [5, 6]. MW ablation results in a deeper tissue penetration and shorter ablation time than RF, leading to a larger ablation volume. The main drawback with MW ablation is the lack of predictability of ablation zone size and shape in less experienced practitioners [7].

High-intensity focused ultrasound (HIFU) or MR-guided focused US or MRgFUS can achieve tissue heating up to $60^{\circ}$ Celsius from absorption of high-intensity focused ultrasonic waves [8]. MRgFUS provides high-resolution guidance and delineation of the target tissue with the added benefit of real-time temperature mapping $[9,10]$. Although the absence of any skin incision is appealing, HIFU is limited to treatment of tumors that can be sonographically visualized and remain unaffected by respiratory motion. It also requires long treatment times, which, in the case of large liver tumors, have been associated with increased incidence of skin burns $[8,11,12]$.

Thermal damage from freezing or cryoablation results from rapid cooling of target tissues. Target temperatures of -20 to $-25{ }^{\circ} \mathrm{C}$ have been shown to induce apoptosis and liquefactive necrosis in animal studies [13]. Cryoablation allows for a decreased need for general anesthesia because of its anesthetic effect. It also allows direct visualization of the ablation margin, which can be useful when performing ablation near critical structures. Lacking any tissue cautery effect, there is an increased risk of bleeding in case of vascular injury and organ fracture due to brittleness of tissue and forceful removal of the ablation probe [14].

\section{Thoracic Interventions}

Lung

Based on projections from the American Cancer Society, 228,190 patients will be diagnosed with lung cancer in 2013, making it the second most common cancer in the US [15]. In patients with early stage primary lung cancer (clinical stage I and II NSCLC) or with limited pulmonary metastatic disease and no extrapulmonary metastases, surgical resection is considered the treatment of choice, with 5-year survival reaching $82 \%$ following lobectomy $[16,17]$.

However, many patients are not considered suitable candidates for surgery because of the unfavorable tumor distribution, number of metastases or high risk comorbidities such as severe chronic obstructive pulmonary disease, a single lung or multiple prior resections limiting the pulmonary reserve and ultimately, leading to poor surgical outcomes. Minimally invasive ablative therapies have emerged as treatment alternatives in patients with smallvolume pulmonary malignancies with contraindications to surgery mainly due to their lesser burden on the pulmonary reserve and high efficacy paralleling those reported in the surgical and radiation therapy literature.

Radiofrequency lung ablation is the most common ablation procedure performed owing to the long-standing presence of RF technology in the medical field. The most favorable evidence toward the use of RF in the treatment of primary and secondary pulmonary malignancies has been the RAPTURE study in 2008. In this large multicenter study, 106 patients with 183 lung tumors deemed unsuitable for surgery, radiotherapy or chemotherapy underwent RF ablation. Overall survival was 70 and $48 \%$ at 1 and 2 years in patients with NSCLC, and 89 and $66 \%$ at 1 and 2 years in patients with colorectal metastases [18]. These findings are further corroborated by the results from Ambrogi et al. [19] who, over the span of 5 years, treated 64 lesions in 54 patients, showing an overall survival of $60 \%$ at 2 years.

In both these studies, metastatic lesions demonstrated better response to treatment compared to primary lung tumors. This is thought to be due to the fact that NSCLC has a tendency for early metastasis making treatment with localized therapies challenging [20].

The rationale for RFA treatment of secondary lung malignancies (Fig. 1) in patients with otherwise controlled primary tumor was based on extensive evidence from the surgical literature where surgical resection has been found to be potentially curative in the event of an isolated lung lesion and can provide increased patient survival by selective metastasectomies and decreasing the tumor burden [21].

Recent studies by Gillams and Petre examined 122 and 45 patients with colorectal lung metastases that were treated with RF ablation. These studies have shown comparable overall survival rates of 57 and $50 \%$ at 3 years in patients [22, 23]. Unsurprisingly, there were more favorable outcomes and better local tumor control in smaller sized tumors. These results were slightly better than those of preceding trials by Yan and Yamakado, which showed 3-year survival rates of 46 and $47 \%$ in a similar patient population $[24,25]$.

Radiofrequency ablation has also been shown to provide an economic advantage over surgical resection in elderly 


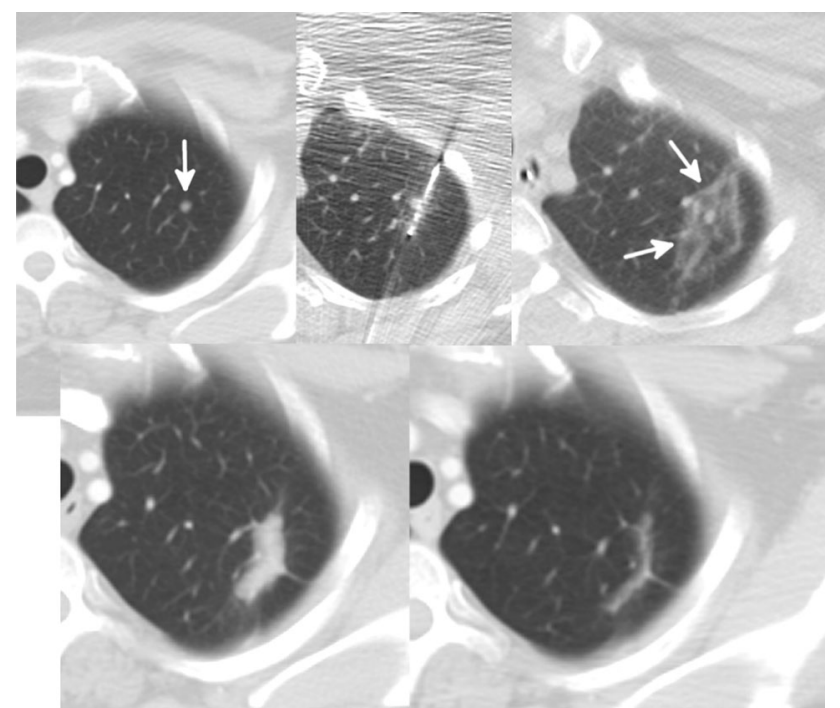

Fig. 1 A 44-year old lady with a history of colorectal cancer who had undergone prior wedge resections presented with a new $1-\mathrm{cm}$ left upper lobe nodule that was increasing in size. She successfully underwent radiofrequency ablation of her left upper lobe mass (top row). Immediate post-ablation scan (top right) shows a typical ground-glass halo surrounding the nodule consistent with the ablation zone. Initial follow-up at 1 month (bottom left) showed post-ablation changes that resolved after 2 years (bottom right) with no evidence of local recurrence

patients in both procedural costs and periprocedural care and hospital stay in a recent study. Although in this study patients treated surgically survived longer, the patients who received RFA treatment were significantly older [26].

The effect of RF ablation on pulmonary function has been found to be negligible in one study [27], and other studies showed recovery in vital capacity and FEV1 to preprocedure values at 3 and 12 months [18, 19]. A decrease in lung function at 1 and 3 months post RFA has been linked to post-procedural pleuritis and the size of the ablation defect. In the majority of cases, complications from pulmonary ablation procedures were primarily related to the placement of needles leading to a higher risk of pneumothorax rather than to complications of the ablative process $[28,29]$.

Microwave ablation of lung tumors has been garnering increased popularity over the last few years owing to some of its advantages over RF ablation. A study on 56 patients with primary and secondary lung metastases further confirmed the safety and efficacy of the MW lung ablation technique. Survival rates of 69,54 and $49 \%$ at 12,24 and 36 months were similar to those found in the RAPTURE trial $[18,30]$.

As mentioned, MW is not affected by the high impedance or low electrical conductivity of lung tissue encountered by RF ablation, and it also has a decreased susceptibility to the heat sink effect from the adjacent large pulmonary vasculature. Although this property allows for potentially larger ablation volumes, tumor size remains an independent predictive factor for local tumor progression in primary and secondary lung cancers, with lesions larger than $1.5 \mathrm{~cm}$ tending to recur [31].

Percutaneous cryoablation of lung tumors has also been applied successfully. In one prospective trial of 71 consecutive patients with 210 lung tumors undergoing percutaneous cryoablation, local progression-free rates achieved were $80.4,69.0$ and $67.7 \%$ at 1,2 and 3 years respectively, though about $95 \%$ of the treated tumors were metastatic, which tend to have a better local success rate. As was also demonstrated in this trial, cryoablation remains affected by the proximity of vessels larger than $3 \mathrm{~mm}$ in a manner comparable to the heat sink effect in RFA [32].

\section{Abdominal Interventions}

\section{Liver}

Liver tumors account for about 30,640 or $1.8 \%$ of the new cancer cases diagnosed in the US in 2013 and for 21,670 estimated deaths in that same year from these cancers [15].

Although surgical resection remains the gold standard for resection of either primary or secondary solitary hepatic tumors with preserved hepatic function [33, 34], there is an emerging role for percutaneous liver ablations for patients with limited tumor burden who are otherwise ineligible for hepatic resection because of poor liver function, comorbid conditions or tumor distribution or as a bridge to hepatic transplantation such as in HCC. Percutaneous ablation can also be used in the "test of time" approach, which consists of performing ablation during the interval between diagnosis and hepatic metastasectomy and monitoring the response in order to limit unnecessary surgery. Patients whose lesions are treated adequately and do not develop additional lesions avoid surgery. Patients whose lesions are not treated adequately but who do not develop additional lesions can still undergo surgery. Patients who develop additional lesions after ablation would no longer be eligible for surgical resection and would have been spared unnecessary hepatectomies [35]. Similar to percutaneous ablations in lungs, hepatic ablation procedures are less invasive than surgery and allow for faster recovery.

Percutaneous RF ablation has been a well-established practice in the treatment of liver tumors, particularly HCC, and has generated a plethora of publications. In 2006, a randomized prospective trial by Chen et al. [36] showed that in 180 patients (of which 71 received RF ablation therapy and 90 were included in the analysis based on intent to treat), RF ablation therapy was found to be as effective as surgical resection in solitary small HCC. 
Overall survival and progression-free survival rates $(\mathrm{OS}=95.8,82.1,71.4$ and $67.9 \%$ and PFS $=85.9,69.3$, 64.1 and $46.4 \%$ at $1,2,3$ and 4 years) were found to be similar to surgical rates with the added advantage of diminished invasiveness. Since then, a more recent study centering on 180 elderly patients older than 65 years of age with early HCC also showed no statistically significant difference in overall survival between the RF ablation group and surgical resection group (93.2, 71.1 and $55.2 \%$ for the RFA group and 88.8, 62.8 and $51.9 \%$ for the surgical group at 1,3 and 5 years, respectively; $P=0.305$ ). What the study did show was that better local control and progression-free survival were achieved for tumors less than $3 \mathrm{~cm}$ in the RFA group in that particular patient population [37]. Use of RF ablation in small HCC tumors was also found to be more cost-effective than surgical resection in patients who have up to three tumors less than $3 \mathrm{~cm}$ in size. However, surgery remains the optimal treatment for larger tumors from the economic standpoint because of better survival rates for a comparatively acceptable increase in cost [38].

In patients with colorectal carcinoma liver metastases, RF ablation has also been extensively studied. Although surgical resection is primarily advocated, there have been reports of survival rates in patients ineligible for surgical resection similar to those achieved by surgical patients. In one study, the overall survival rate was found to be $30 \%$ at 5 years from the time of diagnosis, which was quite close to the reported surgical 5-year survival rate of $32 \%$ [39]. The advantage of having RF ablation technology existing for such a long time is that long-term studies are starting to emerge. A long-term survival study followed 99 patients after undergoing RF ablation of colorectal liver metastases for up to 10 years. These patients were all on systemic chemotherapy and were either ineligible for surgery or had refused surgery. Reported overall survival rates were 98.0, $69.3,47.8,25$ and $18 \%$ at 1, 3, 5, 7 and 10 years, which are close if not comparable to reported survival rates following surgical resection [40•]. Of particular interest was that in the set of patients who developed local recurrences, those that were retreated with RFA had a higher survival rate than those who were not, which illustrates one distinct advantage of the RF ablation technique over surgical resection, which is the fact that RFA is an easily repeatable, minimally invasive procedure. This along with the longterm survival data further supports the potential use of the test-of-time approach in RFA [40•].

Intrahepatic cholangiocarcinoma is relatively rare when compared to HCC or colorectal metastases, but it is also more difficult to treat since most patients present with advanced disease that is unresectable, or, if eligible for surgery, it has the disagreeable tendency to recur following resection. Ablative therapies can be used in these instances to touch up recurrent tumors along the margin [41] or as primary therapy in patients with small tumors who are otherwise not eligible for surgical resection. Five-year survival rates are expectedly lower than in other liver tumors ranging from 20 to $30 \%$ depending on the surgical margin [42, 43]. RF ablations have been performed in small patient series and were relatively successful. Fu et al. [44] showed a 5-year overall survival rate of $28.9 \%$ following RFA in 17 patients with 26 tumors ranging in size from 2.1 to $6.8 \mathrm{~cm}$. Seven of those patients had undergone prior TACE but were unresponsive. Kim et al. [45] treated 13 patients with 17 tumors, achieving local control mainly in tumors smaller than $5 \mathrm{~cm}$ and with a reported 5-year overall survival rate of $15 \%$. It took about 10 years in both these series to gather enough patients, likely owing to the rarity of this type of tumor and to the fact that most unresectable tumors are treated with systemic chemotherapy.

Microwave ablation (Fig. 2) has also become more frequently adopted for hepatic malignancies mainly because of its resistance to the heat sink effect when in proximity to one of the hepatic or portal veins. A multiinstitutional study by Groeschl et al. [46] examined 450 patients who underwent MW ablation for various hepatic malignancies including mainly HCC, colorectal metastases and neuroendocrine tumor metastases. Reported overall survival rates for $\mathrm{HCC}$ and colorectal metastases were 19 and $17 \%$ at 5 years, respectively. These were surprisingly lower than reported survival rates in the RFA literature, despite purported advantages of MW ablation. This is in keeping with findings from a retrospective analysis by Ohmoto et al. [47] that were also in favor of RFA in small HCC tumors, but is contrary to findings from an earlier randomized trial from the same group [48] that showed equivalent results among RF ablation and MW regarding therapeutic effects, complication rates and local control rates. Differences may also be related to patient selection bias in these studies.

Other hepatic tumors such as metastatic GIST or oligonodular neuroendocrine tumors causing symptoms have also been successfully treated with RFA or MWA, and they have demonstrated an improved survival outcome compared to primary liver malignancies or colorectal metastases, but this is likely due to favorable disease histology $[46,49]$.

\section{Kidney}

Renal cancers accounted for 65,150 or $3.9 \%$ of newly diagnosed cancer cases in 2013 in the US [15]. With the refinement of nephron-sparing surgery, partial nephrectomy for early stage renal cell tumors has shown highly successful results, with $77.2 \%$ overall survival at 10 years 


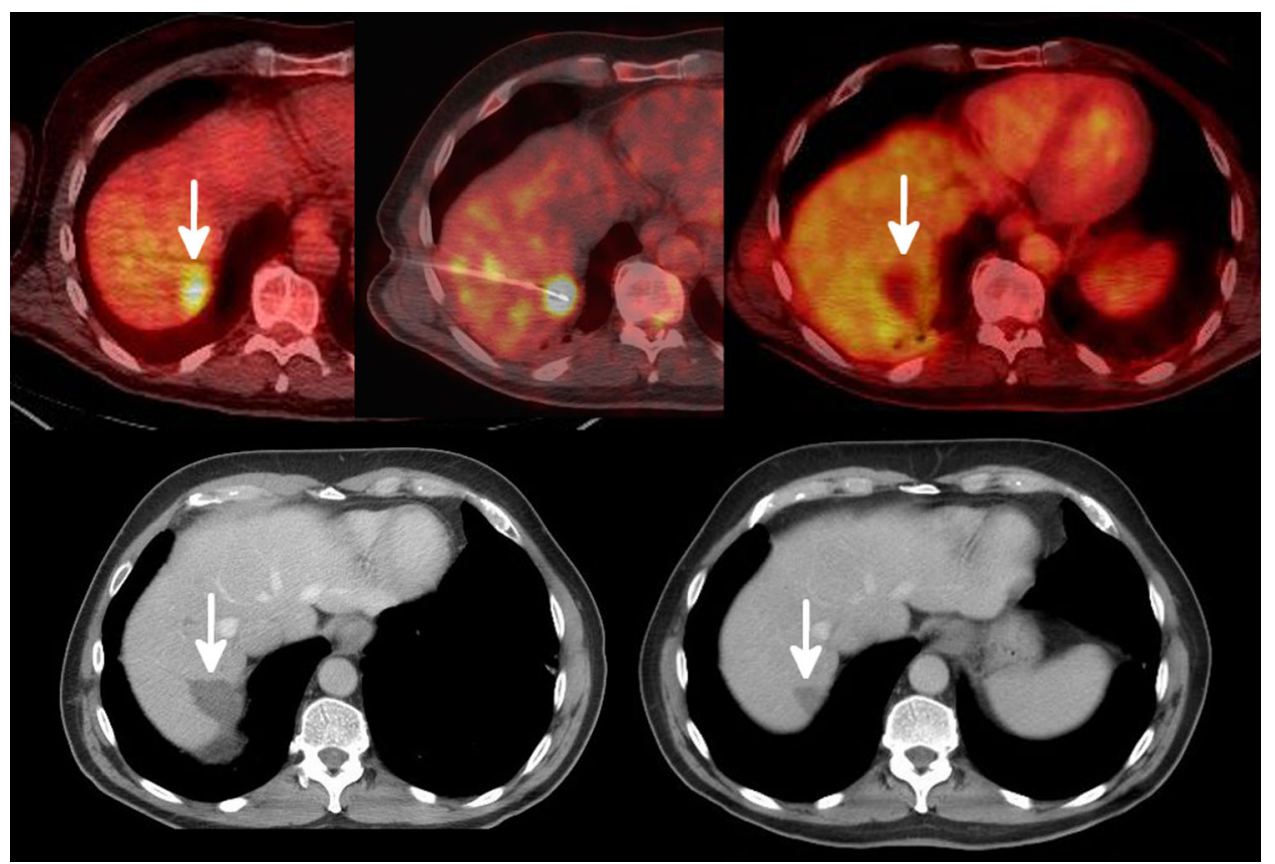

Fig. 2 A 58-year-old man with sigmoid cancer that had been previously resected but later developed liver metastasis that showed up as an FDG-avid lesion in the liver dome on PET-CT (top left). The patient had refused surgery, and so a split-dose PET-CT-guided microwave ablation was successfully performed with no evidence of any residual FDG-avid tumor on the immediate post-procedure PET-

and disease-free survival as high as 97.8 and $95.2 \%$ at 5 and 10 years for T1a tumors [50]. In an otherwise healthy patient with stage 1 tumor, surgical resection remains the standard of care, but thermal ablation is recommended on par with active surveillance in patients with major comorbidities, shortened life expectancy or increased surgical risk [51]. In a recent review of practice patterns in the US, increased age, smaller tumors and recent year of diagnosis were found to correlate with an increased use of thermal ablation for the treatment of stage 1 renal carcinoma [52].

RF ablation has been shown to be a safe and effective treatment modality, and long-term outcomes studies evaluating the treatment of patients with early stage renal cell carcinoma and tumors smaller than $3 \mathrm{~cm}$ have demonstrated high response rates approaching surgical rates [53•, $54 \cdot, 55]$. Two long-term follow-up studies have emerged in the last 2 years. In 2012, Best et al. [53•] reported diseasefree survival rates of 92 and $91 \%$ at 3 and 5 years, but in patients with tumors smaller than $3 \mathrm{~cm}$, rates improved to 96 and $95 \%$. Most recently, Psutka et al. [54•] published the results from their long-term study after following 185 patients with T1 RCC tumors who had undergone RFA. Their disease-free survival rate was $88.6 \%$ at 5 years, but their recurrence-free survival was $95.2 \%$ at 5 years.

Two studies by Takaki et al. [56, 57] compared outcomes by retrospectively reviewing small series of patients
CT scan using a split-dose FDG administration technique [83] (top right). There was no evidence of recurrent disease at his 1-month (bottom left) and 1-year (bottom right) follow-up visits. The scans further showed gradual reduction in the size of his ablation cavity (arrows, bottom row)

with either $\mathrm{T} 1 \mathrm{a}$ or $\mathrm{T} 1 \mathrm{~b}$ renal tumors following either thermal ablation or surgery. Although the overall survival in both studies was found to be lower in the group receiving $\mathrm{RF}$ treatment, it was thought to be due to the clinical background of the patients selected for ablation, who tended to be older and sicker. Adjusted RCC-specific and disease-free survival rates in the RF group in both studies were found to be comparable to surgical outcomes. This was also reflected in further analyses of the outcomes data collected by Choueiri et al. [52] from the SEER Database.

Interestingly, RF ablation need not be restricted to treatment of solid renal tumors. One study of 40 patients [58] showed that RF ablation can also be safely and effectively used in the treatment of complex renal cystic neoplasms such as Bosniak type III and type IV cysts that would have been ideally considered targets for resection in patients with multiple comorbidities precluding them from extensive surgical interventions.

Cryoablation of renal tumors was initially developed for use laparoscopically. Thus, most early studies on cryoablation were comparing laparoscopic and percutaneous approaches to cryoablation and in most instances were found to have similar outcomes with the added benefits for the percutaneous approach of reduced hospital stay and complication rates [59-61]. Although two meta-analyses compared RFA and cryoablation in the treatment of RCC, 
they were limited by the heterogeneity of the studies reviewed, in part because of the inclusion of surgical approaches in addition to percutaneous approaches of cryoablation, and unsurprisingly had differing conclusions $[62,63]$, with one stating that both treatments are equivalent versus another claiming cryoablation is better at local tumor control.

Although cryoablation is generally preferred for larger tumors, a recent retrospective study showed that for renal masses measuring less than $3 \mathrm{~cm}$, both RFA and cryoablation were effectively equivalent treatments. Atwell et al. [64] retrospectively reviewed 385 patients with 445 tumors measuring $3.0 \mathrm{~cm}$ or less treated with either RFA (222 patients) or cryoablation (163 patients). Recurrence-free survival rates at 1,3 and 5 years were $100,98.1$ and $98.1 \%$ for RFA compared to $97.3,90.6$ and $90.6 \%$ for cryoablation. Although differences were noted, they were not statistically significant $(p=0.09)$. This further reaffirms suspected trends in the literature that if differences exist in recurrence-free survival between RFA and cryoablation for early stage small renal tumors, these are likely to be small [65].

Nonetheless, cryoablation continues to gain popularity and garner support in the literature. While most studies up until now have been retrospective, a new prospective study following 134 consecutive patients after cryoablation of stage $1 \mathrm{a} / \mathrm{b}$ RCC tumors showed excellent recurrence-free survival rates of $99.2,98.9$ and $97.0 \%$ at 1,3 and 5 years, which are quite close to the surgical gold standard with the advantage of a more favorable safety profile (Fig. 3) [66].

\section{Pancreas}

The number of new pancreas cancer cases was 45,220 in 2013. The number of deaths related to pancreas cancer is estimated to be 38,460 . Only about $6 \%$ of cases survive by 5 years [15]. Pancreas cancer tends to be aggressive, and patients typically present late in the disease with only about $10 \%$ of tumors confined to the pancreas at the time of presentation. Surgical resection is the preferred treatment, and when thermal ablation is considered, because of the location of the organ and the multiple intervening structures that may hinder a percutaneous approach, ablation is usually performed through an open surgical or laparoscopic approach. However, a few recent pilot studies have evaluated the safety and efficacy of percutaneous approaches to ablation of pancreatic tumors [67-69]. A case report that made it into a small case series of five patients with pancreatic head tumors described treatment with percutaneous MW ablation. Although the series also included five other patients who underwent laparotomy-guided MW ablation, most patients seemed to have tolerated the procedure well
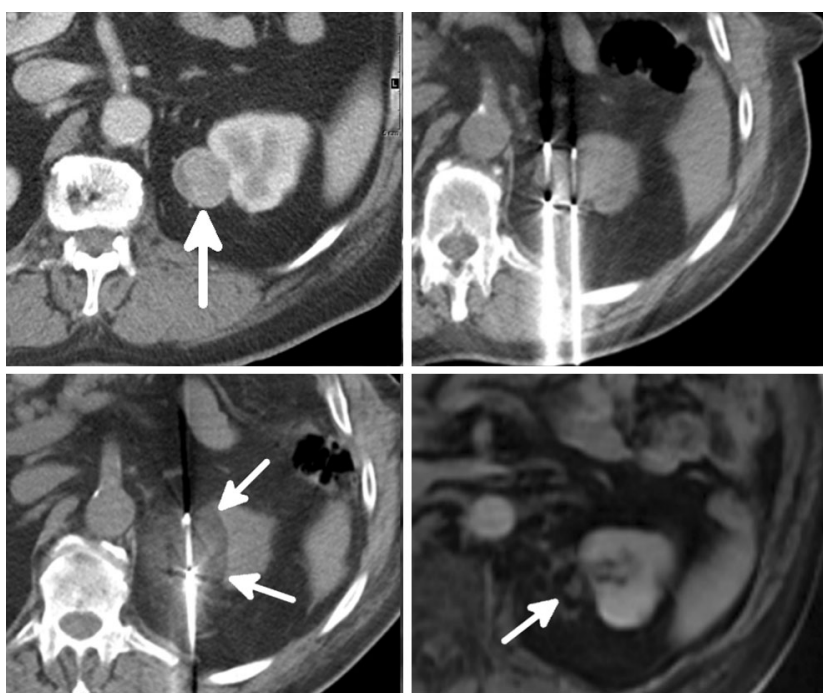

Fig. 3 A 71-year-old man with an incidentally detected, 3-cm left renal mass (top left), which was biopsy proven to be chromophobetype renal cell carcinoma. The patient has several comorbidities including significant coronary artery disease requiring placement of four coronary stents. He opted for non-surgical treatment, and cryoablation (top right) was performed successfully. The procedure was facilitated by visualization of the ablation ice ball on CT (bottom left). On his follow-up visit at 2 years, there was no evidence of recurrence with stable non-enhancing post-ablative changes noted at the site of the previous tumor (bottom right)

with one delayed major complication at 1 month consisting of a pseudoaneurysm of the gastroduodenal artery that was treated using an endovascular approach, although it was not specified from which cohort it originated. Assessment of the treatment's efficacy was limited by the small number of cases and loss to follow-up likely because of the high mortality of this disease [67, 68].

High-intensity focused ultrasound ablation is another ablation modality that has been mainly studied in the treatment of pancreatic cancer because of its distinct advantage of being completely extracorporeal. A pilot study by $\mathrm{Wu}$ et al. [70] in 2005 demonstrated the safety profile of this modality in the palliative treatment of symptomatic advanced pancreatic cancer in the body and tail of the pancreas. Although survival was not necessarily affected, all the patients experienced symptomatic relief from tumor-induced pain. No complications were reported at the time; specifically, no skin burns or pancreatitis were noted. In 2013, a large prospective study evaluated the safety of HIFU on 224 patients with advanced stage III and IV disease. With treatment times limited to less than $2 \mathrm{~h}$, only one patient developed obstructive jaundice 2 weeks after treatment of a lesion in the pancreatic head. Although elevated amylase levels were reported, none of the patients developed symptomatic pancreatitis. Two cases of vertebral injury were identified on MRI but were also asymptomatic. Although the study effectively demonstrated the 
safety of the procedure, it was mainly focused on adverse events, and no data were reported about the efficacy of HIFU in either patient survival or symptom relief. In addition, patients were also excluded if their lesion was undetectable by ultrasound [69]. Additional information as to the therapeutic effectiveness of HIFU ablation in pancreatic tumors is warranted.

Finally, a few studies have been reported on the feasibility of IRE treatment of pancreatic tumors. Based on initial animal and case report studies [71] [72], a small series of 14 patients with unresectable tumors have been treated by IRE. Two patients ultimately underwent surgery and were disease free at 11 and 14 months. Complications included one patient with pancreatitis and one with pneumothorax, and both recovered completely. However, three patients with metastatic disease ultimately died within a short follow-up time [73]. A recent study evaluated the success of tumor ablation in locally advanced pancreatic tumor in 65 patients, but only 12 of those patients underwent percutaneous treatment as opposed to open surgical or laparoscopic treatment. Unsurprisingly, all patients who underwent percutaneous treatment developed local recurrence by 6 months, and although complications were reported, the subgroup in which they occurred was not specified [74].

\section{Advances in Image-Guided Monitoring of Therapy}

The ideal objective of image-guided monitoring of percutaneous ablative therapies is to provide immediate feedback to the interventional radiologist by visually depicting the ablation zone margin and by insuring that adequate coverage of the tumor is achieved and whether there is a need for retreatment. Marginal delineation also insures that the ablation zone does not come too close or in contact with sensitive structures such as nerves, which can lead to irreversible paralysis or paresis. Depending on the target organ, ablation modality and imaging modality, intraprocedural depiction of the ablation zone can be challenging at times. In RF or MW ablation of lung tumors, visualization of a halo of ground-glass attenuation around the ablated nodule on non-enhanced intraprocedural CT scans (Fig. 1) has been found to accurately correlate with the ablation zone margin and corresponds to coagulation necrosis and loss of cellular viability $[75,76]$. Visual changes following RF or MW ablation of liver tumors on non-enhanced CT are less obvious. Although the ablation zone can be seen as a hypoattenuating or heterogeneously hyperattenuating area on non-enhanced CT because of coagulative necrosis and hemorrhage, with or without formation of gas bubbles, the actual margin can be hard to delineate, and occasionally no visible changes may be seen on CT [77]. Use of ultrasound for depiction of the ablation margin is limited to only a few select organs and also by formation of an echogenic cloud and resultant acoustic shadowing, which can persist from $15 \mathrm{~min}$ to $6 \mathrm{~h}$ after ablation [78, 79]. MR thermometry has been used in monitoring ablation zone temperatures by measuring $\mathrm{T} 1$ relaxation times and proton resonance frequency changes. Although mainly developed for use with MRgFUS, it has been applied with RF and MW ablation [10, 80]. Contrast-enhanced CT scans, though useful for long-term follow-up, are limited in the periprocedural period by transient periablational hyperemia that can last up to 1 month and can obscure residual tumor [78]. Although conventional FDG PET was initially thought to be inadequate for monitoring of ablation efficacy due to persistent FDG activity in the ablation zone despite adequate margins $[81,82]$, a new technique using a split-dose method of FDG PET/CT for ablation has shown very promising results. In this study by Ryan et al. [83•], rather than administering the whole FDG dose up front, the standard administered diagnostic FDG dose is split into two aliquots consisting of a small initial dose administered before ablation and used for lesion targeting followed by a larger treatment efficacy dose containing twice the activity of the first dose and administered immediately after the procedure. Therefore, activity from the postablation dose dominates during the acquisition of the postablation images allowing for adequate depiction of the ablation zone as an area of decreased FDG uptake (Fig. 2). Incomplete ablation in this study was demonstrated in one case by a residual focus of increased FDG uptake, which was biopsy proven to represent residual viable tumor, and was immediately retreated. Finally, cryotherapy presents a monitoring advantage over $\mathrm{MW}$ and RF ablation in that the ablation ice ball can be entirely and clearly visualized on both CT and MRI (Fig. 3). Although the ice ball can also be seen on US, the deep margin is obscured by acoustic shadowing, so US monitoring is limited during percutaneous treatment [84].

\section{Conclusion}

In summary, percutaneous image-guided ablative therapy is a viable and accepted option for treatment of early stage tumors in the lung, liver and kidney. It has also shown some indication in the treatment of secondary tumors or in the palliative treatment of advanced tumors. Multiple ablative technologies are available, each with its own recognized advantages and disadvantages, providing increased versatility in treatment with comparable efficacy and similar overall survival rates in some instances. We anticipate that the applicability of ablation technology will expand and become more diverse as the technique and technology 
progresses. As a stand-alone treatment, percutaneous ablation allows for near equivalent results to more invasive treatment options with the advantage of shorter recovery time as well as decreased hospital stay and cost burden.

\section{Compliance with Ethics Guidelines}

Conflict of Interest N. Muallem declares no conflicts of interest. S.B. Solomon has received honoraria for consulting for Covidien, AngioDynamics and GE.

Human and Animal Rights and Informed Consent This article does not contain any studies with human or animal subjects performed by any of the authors.

\section{References}

Papers of particular interest, published recently, have been highlighted as:

- Of importance

1. Lin Z-Z, Shau W-Y, Hsu C, et al. Radiofrequency ablation is superior to ethanol injection in early-stage hepatocellular carcinoma irrespective of tumor size. PLoS One. 2013;8(11):e80276. doi:10.1371/journal.pone.0080276.

2. Maor E, Ivorra A, Leor J, Rubinsky B. The effect of irreversible electroporation on blood vessels. Technol Cancer Res Treat. 2007;6(4):307-12.

3. Al-Sakere B, André F, Bernat C, et al. Tumor ablation with irreversible electroporation. PLoS One. 2007;2(11):e1135. doi:10.1371/journal.pone.0001135.

4. Lencioni R, Crocetti L. Local-regional treatment of hepatocellular carcinoma. Radiology. 2012;262(1):43-58. doi:10.1148/ radiol.11110144.

5. Rhim H, Goldberg SN, Dodd GD, et al. Essential techniques for successful radio-frequency thermal ablation of malignant hepatic tumors. Radiographics. 2001;21:Spec No:S17-35. doi:10.1148/ radiographics.21.suppl_1.g01oc11s17 discussion S36-9.

6. Hong K, Georgiades CS. Radiofrequency ablation: mechanism of action and devices. J Vasc Interv Radiol. 2010;21(8 Suppl):S179-86. doi:10.1016/j.jvir.2010.04.008.

7. Lubner MG, Brace CL, Hinshaw JL, Lee FT. Microwave tumor ablation: mechanism of action, clinical results, and devices. J Vasc Interv Radiol. 2010;21(8 Suppl):S192-203. doi:10.1016/j. jvir.2010.04.007.

8. Zhou Y. High intensity focused ultrasound in clinical tumor ablation. World J Clin Oncol. 2011;2(1):8-27. doi:10.5306/wjco. v2.i1.8.

9. Napoli A, Anzidei M, Ciolina F, et al. MR-guided high-intensity focused ultrasound: current status of an emerging technology. Cardiovasc Intervent Radiol. 2013;36(5):1190-203. doi:10.1007/ s00270-013-0592-4.

10. Quesson B, de Zwart JA, Moonen CT. Magnetic resonance temperature imaging for guidance of thermotherapy. J Magn Reson Imaging. 2000;12(4):525-33.

11. Leslie T, Ritchie R, Illing R, et al. High-intensity focused ultrasound treatment of liver tumours: post-treatment MRI correlates well with intra-operative estimates of treatment volume. $\mathrm{Br} \mathrm{J}$ Radiol. 1018;2012(85):1363-70. doi:10.1259/bjr/56737365.
12. Fischer K, Gedroyc W, Jolesz FA. Focused ultrasound as a local therapy for liver cancer. Cancer J. 2010;16(2):118-24. doi:10. 1097/PPO.0b013e3181db7c32.

13. Hinshaw JL, Lee FT, Laeseke PF, Sampson LA, Brace CL. Temperature isotherms during pulmonary cryoablation and their correlation with the zone of ablation. J Vasc Interv Radiol. 2010;21(9):1424-8. doi:10.1016/j.jvir.2010.04.029.

14. Erinjeri JP, Clark TWI. Cryoablation: mechanism of action and devices. J Vasc Interv Radiol. 2010;21(8 Suppl):S187-91. doi:10. 1016/j.jvir.2009.12.403.

15. Statistical Summaries. Natl Cancer Inst SEER Database. 2013. http://seer.cancer.gov/statfacts/.

16. Baltayiannis N, Chandrinos M, Anagnostopoulos D, et al. Lung cancer surgery: an up to date. J Thorac Dis. 2013;5(Suppl 4):S425-39. doi:10.3978/j.issn.2072-1439.2013.09.17.

17. Villeneuve PJ, Sundaresan RS. Surgical management of colorectal lung metastasis. Clin Colon Rectal Surg. 2009;22(4):233-41. doi:10.1055/s-0029-1242463.

18. Lencioni R, Crocetti L, Cioni R, et al. Response to radiofrequency ablation of pulmonary tumours: a prospective, intention-to-treat, multicentre clinical trial (the RAPTURE study). Lancet Oncol. 2008;9(7):621-8. doi:10.1016/S14702045(08)70155-4.

19. Ambrogi MC, Lucchi M, Dini P, et al. Percutaneous radiofrequency ablation of lung tumours: results in the mid-term. Eur $\mathrm{J}$ Cardiothorac Surg. 2006;30(1):177-83. doi:10.1016/j.ejcts.2006. 03.067.

20. Donington J, Ferguson M, Mazzone P, et al. American College of Chest Physicians and Society of Thoracic Surgeons consensus statement for evaluation and management for high-risk patients with stage I non-small cell lung cancer. Chest. 2012;142(6):1620-35. doi:10.1378/chest.12-0790.

21. Kaifi JT, Gusani NJ, Deshaies I, et al. Indications and approach to surgical resection of lung metastases. J Surg Oncol. 2010;102(2):187-95. doi:10.1002/jso.21596.

22. Gillams AR, Khan Z, Osborn P, Lees WR. Survival after radiofrequency ablation in 122 patients with inoperable colorectal lung metastases. Cardiovasc Intervent Radiol. 2013;36(3):724-30. doi:10.1007/s00270-012-0500-3.

23. Petre EN, Jia X, Thornton RH, et al. Treatment of pulmonary colorectal metastases by radiofrequency ablation. Clin Colorectal Cancer. 2013;12(1):37-44. doi:10.1016/j.clcc.2012.07.003.

24. Yan TD, King J, Sjarif A, Glenn D, Steinke K, Morris DL. Percutaneous radiofrequency ablation of pulmonary metastases from colorectal carcinoma: prognostic determinants for survival. Ann Surg Oncol. 2006;13(11):1529-37. doi:10.1245/s10434-0069101-1.

25. Yamakado K, Hase S, Matsuoka T, et al. Radiofrequency ablation for the treatment of unresectable lung metastases in patients with colorectal cancer: a multicenter study in Japan. J Vasc Interv Radiol. 2007;18(3):393-8. doi:10.1016/j.jvir.2006.11.003.

26. Alexander ES, Machan JT, Ng T, Breen LD, DiPetrillo TA, Dupuy DE. Cost and effectiveness of radiofrequency ablation versus limited surgical resection for stage I non-small-cell lung cancer in elderly patients: is less more? J Vasc Interv Radiol. 2013;24(4):476-82. doi:10.1016/j.jvir.2012.12.016.

27. De Baère T, Palussière $J$, Aupérin A, et al. Midterm local efficacy and survival after radiofrequency ablation of lung tumors with minimum follow-up of 1 year: prospective evaluation. Radiology. 2006;240(2):587-96. doi:10.1148/radiol.2402050807.

28. Tada A, Hiraki T, Iguchi T, et al. Influence of radiofrequency ablation of lung cancer on pulmonary function. Cardiovasc Intervent Radiol. 2012;35(4):860-7. doi:10.1007/s00270-011-0221-z.

29. Baisi A, De Simone M, Raveglia F, Cioffi U. Thermal ablation in the treatment of lung cancer: present and future. Eur J Cardiothorac Surg. 2013;43(4):683-6. doi:10.1093/ejcts/ezs558. 
30. Belfiore G, Ronza F, Belfiore MP, et al. Patients' survival in lung malignancies treated by microwave ablation: our experience on 56 patients. Eur J Radiol. 2013;82(1):177-81. doi:10.1016/j. ejrad.2012.08.024.

31. Vogl TJ, Worst TS, Naguib NN, Ackermann H, Gruber-Rouh T, Nour-Eldin NE. Factors influencing local tumor control in patients with neoplastic pulmonary nodules treated with microwave ablation: a risk-factor analysis. AJR Am J Roentgenol. 2013;200(3):665-72. doi:10.2214/AJR.12.8721.

32. Yashiro H, Nakatsuka S, Inoue $M$, et al. Factors affecting local progression after percutaneous cryoablation of lung tumors. J Vasc Interv Radiol. 2013;24(6):813-21. doi:10.1016/j.jvir.2012. 12.026 .

33. Arii S, Yamaoka Y, Futagawa S, et al. Results of surgical and nonsurgical treatment for small-sized hepatocellular carcinomas: a retrospective and nationwide survey in Japan. The Liver Cancer Study Group of Japan. Hepatology. 2000;32(6):1224-9. doi:10. 1053/jhep.2000.20456.

34. Ochiai T, Ohta K, Iida M, et al. High resectability of colorectal liver metastases with aggressive chemotherapy in the era of molecular target-based agents. Hepatogastroenterology. 2013;60(125):955-60. doi:10.5754/hge121213.

35. Livraghi T, Solbiati L, Meloni MF, Ierace T, Goldberg SN, Gazelle GS. Percutaneous radiofrequency ablation of liver metastases in potential candidates for resection: the "test-of-time approach". Cancer. 2003;97(12):3027-35. doi:10.1002/cncr. 11426.

36. Chen M-S, Li J-Q, Zheng Y, et al. A prospective randomized trial comparing percutaneous local ablative therapy and partial hepatectomy for small hepatocellular carcinoma. Ann Surg. 2006;243(3):321-8. doi:10.1097/01.sla.0000201480.65519.b8.

37. Peng Z-W, Liu F-R, Ye $S$, et al. Radiofrequency ablation versus open hepatic resection for elderly patients ( $>65$ years) with very early or early hepatocellular carcinoma. Cancer. 2013;119(21):3812-20. doi:10.1002/cncr.28293.

38. Cucchetti A, Piscaglia F, Cescon M, et al. Cost-effectiveness of hepatic resection versus percutaneous radiofrequency ablation for early hepatocellular carcinoma. J Hepatol. 2013;59(2):300-7. doi:10.1016/j.jhep.2013.04.009.

39. Gillams AR, Lees WR. Radio-frequency ablation of colorectal liver metastases in 167 patients. Eur Radiol. 2004;14(12):2261-7. doi:10.1007/s00330-004-2416-z.

40. - Solbiati L, Ahmed M, Cova L, Ierace T, Brioschi M, Goldberg SN. Small liver colorectal metastases treated with percutaneous radiofrequency ablation: local response rate and long-term survival with up to 10-year follow-up. Radiology. 2012;265(3):958-68. doi:10.1148/radiol.12111851. This reference is of importance because it provides long-term survival data following RF ablation treatment of liver metastases.

41. Kim JH, Won HJ, Shin YM, Kim PN, Lee S-G, Hwang S. Radiofrequency ablation for recurrent intrahepatic cholangiocarcinoma after curative resection. Eur J Radiol. 2011;80(3):e221-5. doi:10.1016/j.ejrad.2010.09.019.

42. Guglielmi A, Ruzzenente A, Campagnaro T, et al. Intrahepatic cholangiocarcinoma: prognostic factors after surgical resection. World J Surg. 2009;33(6):1247-54. doi:10.1007/s00268-0099970-0.

43. Lang H, Sotiropoulos GC, Sgourakis G, et al. Operations for intrahepatic cholangiocarcinoma: single-institution experience of 158 patients. J Am Coll Surg. 2009;208(2):218-28. doi:10.1016/j. jamcollsurg.2008.10.017.

44. Fu Y, Yang W, Wu W, Yan K, Xing BC, Chen MH. Radiofrequency ablation in the management of unresectable intrahepatic cholangiocarcinoma. J Vasc Interv Radiol. 2012;23(5):642-9. doi:10.1016/j.jvir.2012.01.081.
45. Kim JH, Won HJ, Shin YM, Kim K-A, Kim PN. Radiofrequency ablation for the treatment of primary intrahepatic cholangiocarcinoma. AJR Am J Roentgenol. 2011;196(2):W205-9. doi:10. 2214/AJR.10.4937.

46. Groeschl RT, Pilgrim CHC, Hanna EM, et al. Microwave ablation for hepatic malignancies: a multiinstitutional analysis. Ann Surg. 2013;00(00):1-6. doi:10.1097/SLA.0000000000000234.

47. Ohmoto K, Yoshioka N, Tomiyama Y, et al. Comparison of therapeutic effects between radiofrequency ablation and percutaneous microwave coagulation therapy for small hepatocellular carcinomas. J Gastroenterol Hepatol. 2009;24(2):223-7. doi:10. 1111/j.1440-1746.2008.05596.x.

48. Shibata T, Iimuro Y, Yamamoto Y, et al. Small hepatocellular carcinoma: comparison of radio-frequency ablation and percutaneous microwave coagulation therapy. Radiology. 2002;223(2):331-7. doi:10.1148/radiol.2232010775.

49. Yamanaka T, Takaki H, Nakatsuka A, et al. Radiofrequency ablation for liver metastasis from gastrointestinal stromal tumor. J Vasc Interv Radiol. 2013;24(3):341-6. doi:10.1016/j.jvir.2012.11.021.

50. Lane BR, Campbell SC, Gill IS. 10-Year oncologic outcomes after laparoscopic and open partial nephrectomy. J Urol. 2013;190(1):44-9. doi:10.1016/j.juro.2012.12.102.

51. Campbell SC, Novick AC, Belldegrun A, et al. Guideline for management of the clinical T1 renal mass. J Urol. 2009;182(4):1271-9. doi:10.1016/j.juro.2009.07.004.

52. Choueiri TK, Schutz FA, Hevelone ND, et al. Thermal ablation vs surgery for localized kidney cancer: a Surveillance, Epidemiology, and End Results (SEER) database analysis. Urology. 2011;78(1):93-8. doi:10.1016/j.urology.2011.01.068.

53. - Best SL, Park SK, Youssef RF, et al. Long-term outcomes of renal tumor radio frequency ablation stratified by tumor diameter: size matters. J Urol. 2012;187(4):1183-9. doi:10.1016/j.juro. 2011.11.096. A good paper about the long-term survival data following RF ablation treatment of renal tumors.

54. • Psutka SP, Feldman AS, McDougal WS, McGovern FJ, Mueller PR, Gervais DA. Long-term oncologic outcomes after radiofrequency ablation for $\mathrm{T} 1$ renal cell carcinoma. Eur Urol. 2013;63(3):486-92. doi:10.1016/j.eururo.2012.08.062. A good paper about the long-term survival data following $R F$ ablation treatment of renal tumors.

55. Gervais DA, McGovern FJ, Arellano RS, McDougal WS, Mueller PR. Radiofrequency ablation of renal cell carcinoma: part 1, indications, results, and role in patient management over a 6-year period and ablation of 100 tumors. AJR Am J Roentgenol. 2005;185(1):64-71. doi:10.2214/ajr.185.1.01850064.

56. Takaki H, Yamakado K, Soga N, et al. Midterm results of radiofrequency ablation versus nephrectomy for T1a renal cell carcinoma. Jpn J Radiol. 2010;28(6):460-8. doi:10.1007/s11604010-0451-z.

57. Takaki H, Soga N, Kanda H, et al. Radiofrequency ablation versus radical nephrectomy: clinical outcomes for stage $\mathrm{T} 1 \mathrm{~b}$ renal cell carcinoma. Radiology. 2014;270(1):292-9. doi:10.1148/ radiol.13130221.

58. Allen BC, Chen MY, Childs DD, Zagoria RJ. Imaging-guided radiofrequency ablation of cystic renal neoplasms. AJR Am J Roentgenol. 2013;200(6):1365-9. doi:10.2214/AJR.12.9336.

59. Long CJ, Kutikov A, Canter DJ, et al. Percutaneous vs surgical cryoablation of the small renal mass: is efficacy compromised? BJU Int. 2011;107(9):1376-80. doi:10.1111/j.1464-410X.2010.09851.x.

60. Goyal J, Verma P, Sidana A, Georgiades CS, Rodriguez R. Single-center comparative oncologic outcomes of surgical and percutaneous cryoablation for treatment of renal tumors. J Endourol. 2012;26(11):1413-9. doi:10.1089/end.2012.0244.

61. Mues AC, Okhunov Z, Haramis G, D'Agostino HB, Shingleton BW, Landman J. Comparison of percutaneous and laparoscopic 
renal cryoablation for small $(<3.0 \mathrm{~cm})$ renal masses. J Endourol. 2010;24(7):1097-100. doi:10.1089/end.2010.0067.

62. El Dib R, Touma NJ, Kapoor A. Cryoablation vs radiofrequency ablation for the treatment of renal cell carcinoma: a meta-analysis of case series studies. BJU Int. 2012;110(4):510-6. doi:10.1111/j. 1464-410X.2011.10885.x.

63. Kunkle DA, Uzzo RG. Cryoablation or radiofrequency ablation of the small renal mass: a meta-analysis. Cancer. 2008;113(10):2671-80. doi:10.1002/cncr.23896.

64. Atwell TD, Schmit GD, Boorjian SA, et al. Percutaneous ablation of renal masses measuring $3.0 \mathrm{~cm}$ and smaller: comparative local control and complications after radiofrequency ablation and cryoablation. AJR Am J Roentgenol. 2013;200(2):461-6. doi:10. 2214/AJR.12.8618.

65. Gervais DA. Cryoablation versus radiofrequency ablation for renal tumor ablation: time to reassess? J Vasc Interv Radiol. 2013;24(8):1135-8. doi:10.1016/j.jvir.2013.05.030.

66. Georgiades CS, Rodriguez R. Efficacy and safety of percutaneous cryoablation for stage 1A/B renal cell carcinoma: results of a prospective, single-arm, 5-year study. Cardiovasc Intervent Radiol. 2014;. doi:10.1007/s00270-013-0831-8.

67. Carrafiello G, Ierardi AM, Piacentino F, et al. Microwave ablation with percutaneous approach for the treatment of pancreatic adenocarcinoma. Cardiovasc Intervent Radiol. 2012;35(2):439-42. doi:10.1007/s00270-011-0189-8.

68. Carrafiello G, Ierardi AM, Fontana F, et al. Microwave ablation of pancreatic head cancer: safety and efficacy. J Vasc Interv Radiol. 2013;24(10):1513-20. doi:10.1016/j.jvir.2013.07.005.

69. Wang K, Zhu H, Meng Z, et al. Safety evaluation of highintensity focused ultrasound in patients with pancreatic cancer. Onkologie. 2013;36(3):88-92. doi:10.1159/000348530.

70. Wu F, Wang Z-B, Zhu H, et al. Feasibility of US-guided highintensity focused ultrasound treatment in patients with advanced pancreatic cancer: initial experience. Radiology. 2005;236(3):1034-40. doi:10.1148/radiol.2362041105.

71. Bower M, Sherwood L, Li Y, Martin RCG. Irreversible electroporation of the pancreas: definitive local therapy without systemic effects. J Surg Oncol. 2011;104(1):22-8. doi:10.1002/jso. 21899.

72. Bagla S, Papadouris D. Percutaneous irreversible electroporation of surgically unresectable pancreatic cancer: a case report. J Vasc Interv Radiol. 2012;23(1):142-5. doi:10.1016/j.jvir.2011.10.002.

73. Narayanan G, Hosein PJ, Arora G, et al. Percutaneous irreversible electroporation for downstaging and control of unresectable pancreatic adenocarcinoma. J Vasc Interv Radiol. 2012;23(12):1613-21. doi:10.1016/j.jvir.2012.09.012.

74. Dunki-Jacobs EM, Philips P, Martin RCG. Evaluation of resistance as a measure of successful tumor ablation during irreversible electroporation of the pancreas. J Am Coll Surg. 2014;218(2):179-87. doi:10.1016/j.jamcollsurg.2013.10.013.

75. Goldberg SN, Gazelle GS, Compton CC, McLoud TC. Radiofrequency tissue ablation in the rabbit lung: efficacy and complications. Acad Radiol. 1995;2(9):776-84.

76. Kuroki M, Nakada H, Yamashita A, et al. Loss of cellular viability in areas of ground-glass opacity on computed tomography images immediately after pulmonary radiofrequency ablation in rabbits. Jpn J Radiol. 2012;30(4):323-30. doi:10.1007/s11604012-0054-y.

77. Sainani NI, Gervais DA, Mueller PR, Arellano RS. Imaging after percutaneous radiofrequency ablation of hepatic tumors: part 1, normal findings. AJR Am J Roentgenol. 2013;200(1):184-93. doi:10.2214/AJR.12.8478.

78. Kim Y, Rhim H, Lim HK, Choi D, Lee MW, Park MJ. Coagulation necrosis induced by radiofrequency ablation in the liver: histopathologic and radiologic review of usual to extremely rare changes. Radiographics. 2011;31(2):377-90. doi:10.1148/rg. 312105056.

79. Choi D, Lim HK, Kim SH, et al. Hepatocellular carcinoma treated with percutaneous radio-frequency ablation: usefulness of power Doppler US with a microbubble contrast agent in evaluating therapeutic response-preliminary results. Radiology. 2000;217(2):558-63. doi:10.1148/radiology.217.2.r00oc07558.

80. De Senneville BD, Mougenot C, Quesson B, Dragonu I, Grenier $\mathrm{N}$, Moonen CTW. MR thermometry for monitoring tumor ablation. Eur Radiol. 2007;17(9):2401-10. doi:10.1007/s00330-0070646-6.

81. Schoellnast H, Larson SM, Nehmeh SA, Carrasquillo JA, Thornton RH, Solomon SB. Radiofrequency ablation of nonsmall-cell carcinoma of the lung under real-time FDG PET CT guidance. Cardiovasc Intervent Radiol. 2011;2011(34 Suppl 2):S182-5. doi:10.1007/s00270-010-9898-7.

82. Sainani NI, Shyn PB, Tatli S, Morrison PR, Tuncali K, Silverman SG. PET/CT-guided radiofrequency and cryoablation: is tumor fluorine-18 fluorodeoxyglucose activity dissipated by thermal ablation? J Vasc Interv Radiol. 2011;22(3):354-60. doi:10.1016/ j.jvir.2010.11.027.

83. - Ryan ER, Sofocleous CT, Schöder H, et al. Split-dose technique for FDG PET/CT-guided percutaneous ablation: a method to facilitate lesion targeting and to provide immediate assessment of treatment effectiveness. Radiology. 2013;268(1):288-95. doi:10. 1148/radiol.13121462. This reference is of importance because it provides a new technique that allows the use of FDG PET in monitoring ablation efficacy.

84. Mala T, Aurdal L, Frich L, et al. Liver tumor cryoablation: a commentary on the need of improved procedural monitoring. Technol Cancer Res Treat. 2004;3(1):85-91. 